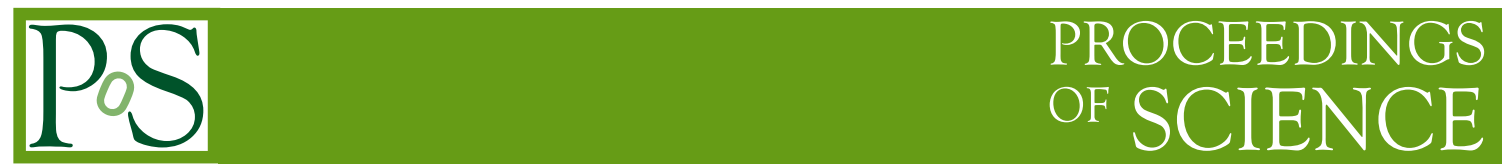

\title{
Continuum Thermodynamics of the SU(N) Gauge Theory
}

\section{Saumen Datta* and Sourendu Gupta}

Tata Institute of Fundamental Research

E-mail: saumen@theory.tifr.res.in, sgupta@tifr.res.in

The thermodynamics of the deconfined phase of the SU(N) gauge theory is studied. Careful study is made of the approach to the continuum limit. The latent heat of the deconfinement transition is studied, for the theories with 3, 4 and 6 colors. Continuum estimates of various thermodynamic quantities are studied, and the approach to conformality investigated. The bulk thermodynamic quantities at different $\mathrm{N}$ are compared, to investigate the validity of 'tHooft scaling at these values of $\mathrm{N}$.

The XXVIII International Symposium on Lattice Filed Theory

June 14-19,2010

Villasimius, Sardinia Italy

\footnotetext{
*Speaker.
} 


\section{Introduction}

Interest in $\mathrm{SU}(\mathrm{N})$ gauge theories with large $\mathrm{N}$ began with the pioneering studies of [1], who showed that in the limit $\mathrm{N} \rightarrow \infty$ and the gauge coupling, $g \rightarrow 0$, with the 't Hooft coupling $\lambda=g^{2} \mathrm{~N}$ fixed, one gets a non-trivial but simplified theory. Many qualitative features of hadron physics can be explained by appealing to this limit.

The theory with an infinite number of colors has also been used to understand various facets of the phase diagram of strongly interacting matter. At very high temperatures, strongly interacting matter is known to exist in a deconfined, chirally symmetric state. The nature of the transition to this state is quite sensitive to the quark sector. For infinitely massive quarks, one has a first order deconfinement transition for $\mathrm{N}>2$. For two massless quarks, on the other hand, one has a second order, chiral symmetry restoring transition. If a small mass is given to the quarks, one expects a crossover, and a critical point at some nonzero baryon density. See Ref. [2] for a detailed discussion of the nature of the transition. A rich phase structure has also been predicted for the large baryon density regime. In particular, a chirally symmetric, confined phase has been predicted, using the 't Hooft limit [3].

For phase diagrams based on arguments about the theory with an infinite number of colors to be relevant for the theory with three colors, it is important to explore the validity of the large $\mathrm{N}$ arguments for $\mathrm{N}=3$. Analogies have also been drawn between the high temperature phase of QCD and the solvable, conformal $\mathscr{N}=4$ supersymmetric $\mathrm{SU}(\mathrm{N} \rightarrow \infty)$ theory [4]. Lattice studies of the theory with moderate values of $\mathrm{N}$ can provide one intermediate step in this connection, by giving an estimate of the size of the $1 / \mathrm{N}^{2}$ corrections.

In this report, we present results for a study of the thermodynamics of the $\mathrm{SU}(\mathrm{N})$ gauge theories at finite temperatures for $\mathrm{N}=4,6$, and combine them with results for $\mathrm{N}=3$ to get an estimate of the applicability of the large $\mathrm{N}$ arguments for the theory with three colors. For $\mathrm{N}=3$, we have used the simulation results of Ref. [5], supplementing them with new simulations where necessary (in particular, for the measurement of the latent heat), and analyzed them using the same techniques as used for the $\mathrm{N}=4$ and 6 theories. For estimating the thermodynamic quantities, we use the standard methods [5]. The pressure, $\mathrm{p}$, and $\Delta=\varepsilon-3 p$, where $\varepsilon$ is the energy density, are calculated from the plaquette data,

$$
\frac{\Delta}{T^{4}}=6 N_{t}^{4} \frac{\partial \beta}{\partial \log a} \delta P(\beta, T), \quad \frac{p(T)}{T^{4}}-\frac{p\left(T_{0}\right)}{T_{0}^{4}}=6 N_{t}^{4} \int_{\beta_{0}}^{\beta} d \beta \delta P(\beta, T)
$$

where $\delta P(\beta, T)=P(\beta, T)-P(\beta, T=0)$ is the difference in the plaquette observables between the finte temperature lattice and the corresponding zero temperature lattice, calculated at the coupling $\beta . T_{0}$ is some reference temperature. $\partial \beta / \partial \log a$ is related to the beta function. We use a nonperturbative estimate for the beta function, using the scaling of $T_{c}$ from [6]. Details of the beta function used by us can be found in [2].

In the next section we discuss the latent heat of the deconfinement transition for the theory with $\mathrm{N}=3,4,6$ number of colors. In Sec. 3 we study the equation of state of the SU(N) gluon plasma. A comparison of the energy density and pressure for $\mathrm{SU}(3,4,6)$ gives us an idea of the size of the $1 / \mathrm{N}^{2}$ corrections. In the final section we discuss the physical implications of our results. 
$\mathrm{SU}(\mathrm{N})$ gauge theories have been investigated previously on the lattice. In particular, the latent heat of the transition has been studied in Ref. [7, 8], while the equation of state has been studied in Ref. [9, 10]. The focus of our work is in getting results for the continuum limit. We use a nonperturbative beta function, and multiple lattice spacings at each temperature, for this purpose. We have also employed several spatial volumes at each lattice spacing, to reach the thermodynamic limit. Details of our work, including the cutoff and volume dependence, can be obtained in Ref. [2]. The results reported here are our estimates of the infinite volume, continuum results. An earlier version of our study, which used the two-loop beta function, and had measurements at fewer temperatures, was presented in the previous year's conference [11].

\section{Latent Heat}

$\mathrm{SU}(\mathrm{N})$ gauge theories have a first order deconfinement transition for $\mathrm{N} \geq 3$. A first order transition is characterized by a latent heat associated with the transition. For the infinite volume system, the latent heat can be defined as

$$
\frac{L_{h}}{T_{c}^{4}}=\lim _{\delta T \rightarrow 0}\left(\frac{\varepsilon\left(T_{c}+\delta T\right)}{T_{c}^{4}}-\frac{\varepsilon\left(T_{c}-\delta T\right)}{T_{c}^{4}}\right)=\lim _{\delta T \rightarrow 0}\left(\frac{\Delta\left(T_{c}+\delta T\right)}{T_{c}^{4}}-\frac{\Delta\left(T_{c}-\delta T\right)}{T_{c}^{4}}\right)
$$

where $\varepsilon\left(T_{c} \pm \delta T\right)$ are the energy densities of the confined and deconfined phases, respectively, and the second equality follows from the fact that pressure is continuous across the transition.

For a finite volume system, as is necessarily used in a numerical lattice computation, one cannot get a separation of the phases, and so, a straightforward $\delta T \rightarrow 0$ limit will not work. We use the following method to extract the latent heat [2]. Since the confined and the deconfined phases are resolved by the Polyakov loop $|L|$, we identify the configurations at the transition point with $|L|<L_{c}$ as being in the confined phase and those with $|L|>L_{h}$ as being in the deconfined phase, where $L_{c}$ and $L_{h}$ are suitably chosen values. In order for the procedure to be meaningful and not too sensitive on the choice of $L_{h}$ and $L_{c}$, it is important that $|L|$ shows a two-peak structure. Figure 1 (a) shows the distribution of $|L|$ in the transition regime of SU(4) gauge theory. $L_{c}$ and $L_{h}$ are chosen at the valley (see Ref. [2] for details). The distribution of $\delta P(T)$ for the two phases defined by the $|L|$ cut is quite stable, as shown in Fig. 1 (b): for values of the coupling $\beta$ in the transition regime, the histograms for $\delta P(T)$ for the two phases defined by the $|L|$ cut essentially coincide.

From the histograms of $\delta P(T)$ for the two phases at $T_{c}$, the latent heat can be calculated using Eq.(2.1) and Eq.(1.1):

$$
\frac{L_{h}}{T_{c}^{4}}=6 N_{t}^{4} \frac{\partial \beta}{\partial \log a}\left(P\left(T_{c}^{+}\right)-P\left(T_{c}^{-}\right)\right)
$$

where, as mentioned in the introduction, $\partial \beta / \partial \log a$ is evaluated from the nonperturbative scalesetting in [6]. We find that the finite volume effects in the latent heat are rather large for SU(3), where one needs an aspect ratio $\zeta=L T>6$ to reach the thermodynamic limit. On the other hand, for $\mathrm{SU}(4)$ and $\mathrm{SU}(6)$ gauge theories an aspect ratio $\zeta \geq 3$ suffices to reach the thermodynamic limit. This is probably related to the large correlation length of the Polyakov loop for SU(3) gauge theory near the transition. Our estimates for the thermodynamic limits of the latent heat for the theories with $\mathrm{N}=3,4$ and 6 are shown in Table 1 . To estimate the sensitivity of the results on the 

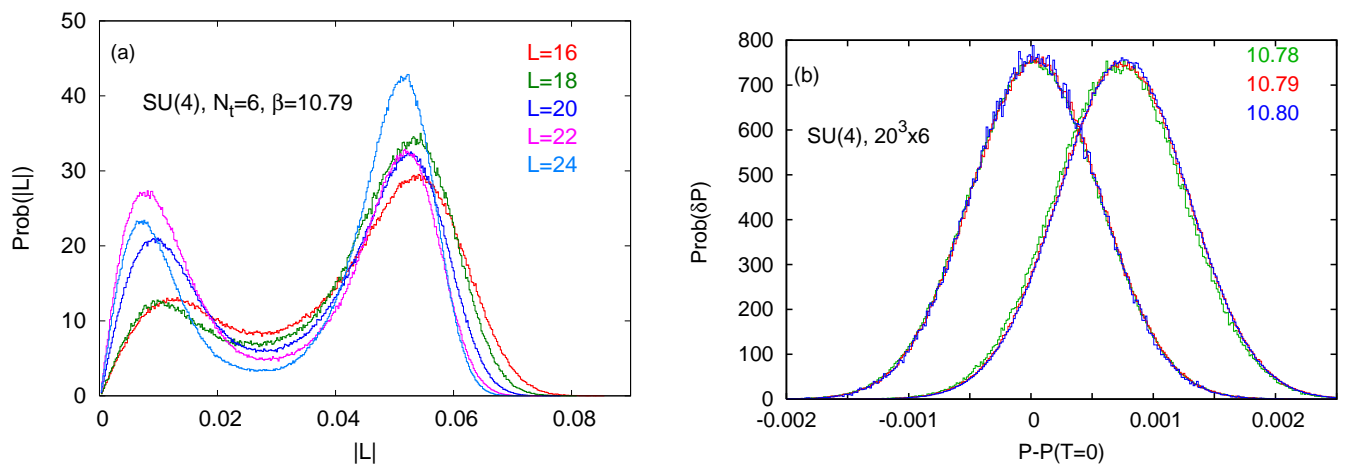

Figure 1: (a) Distribution of the Polyakov loop, $|\mathrm{L}|$, in the transition region for SU(4) gauge theory. (b) Distribution of the plaquette, $P(T)-P(T=0)$, for the cold and hot phases, identified by configurations with $|L|<L_{c}$ and $|L|>L_{h}$, respectively.

Table 1: Latent heat for $\mathrm{SU}(\mathrm{N})$ gauge theories with $\mathrm{N}=3,4$ and 6 . The numbers in brackets are the errors on the least significant digits; the first one is statistical and the second, systematic.

\begin{tabular}{c|ccc}
\hline $\mathrm{N}$ & 3 & 4 & 6 \\
$L_{h} / T_{c}^{4}$ & $1.67(4)(4)$ & $4.32(6)(6)$ & $11.93(34)(5)$ \\
\hline
\end{tabular}

choices of $L_{c, h}$, we vary $L_{h}-L_{c}$ by $\pm 20 \%$; the corresponding change in the latent heat is shown as a systematic error in the table.

Our results for the latent heat are in good agreement with Ref. [8], within the larger uncertainties of that study. The values obtained by Ref. [7] are somewhat higher. On going from $\mathrm{N}=3$ to 4 , the latent heat is seen to scale faster than the Casimir $d_{A}=\mathrm{N}^{2}-1$, the dimensionality of the adjoint representation. On the other hand, the scaling between $\mathrm{N}=4$ and 6 is consistent with a Casimir scaling. Taking all the three values in the Table, one can get a good fit to the relation

$$
\frac{L_{h}}{d_{A} T_{c}^{4}}=0.388(3)-\frac{1.61(4)}{\mathrm{N}^{2}}
$$

where the errors are statistical only. Eq.(2.3) indicates a large correction to the leading term for the theory with three colors.

\section{Equation of State}

We first discuss $\Delta$, which is the trace of the energy-momentum tensor and therefore is a measure of conformal symmetry breaking. Fig. 2 shows $\Delta$ for $\mathrm{SU}(\mathrm{N})$ with different number of colors, measured at different temperatures, and normalised by $d_{A} T^{4}$.

As the figure shows, $\Delta$ scales nicely with $d_{A}$ except very close to $T_{c}$. The size of the $1 / \mathrm{N}^{2}$ correction is smaller than the statistical accuracy of our data, for $T>1.25 T_{c}$. Closer to $T_{c}$ we see a deviation from scaling with $d_{A}$. The peak of $\Delta$ is seen to become higher and move closer to $T_{c}$ with increasing N. At higher temperatures, $\Delta$ scales approximately like $T^{2}$ [12], for the temperature range investigated by us [2]. 


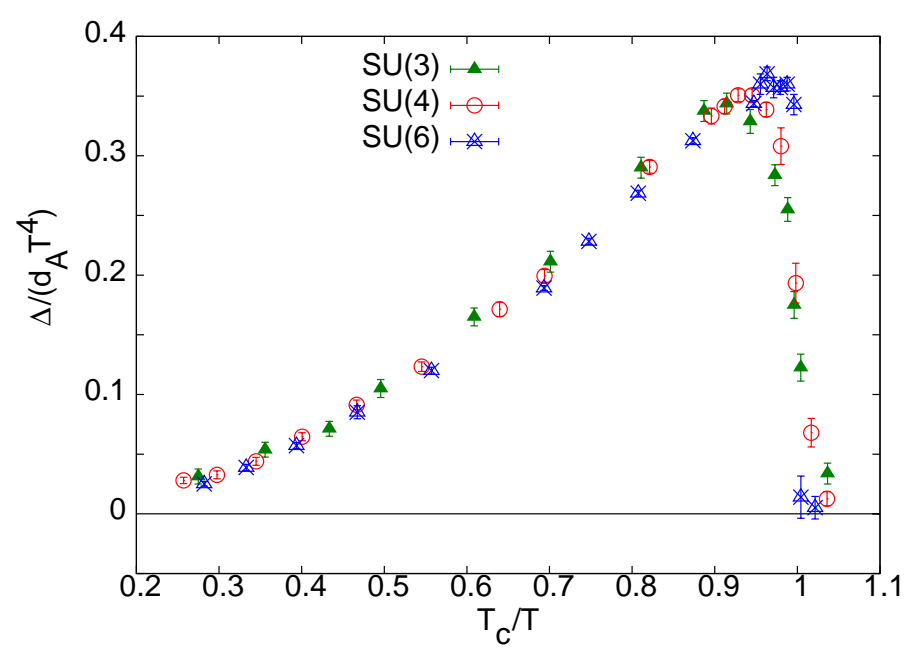

Figure 2: $\Delta / d_{A} T^{4}$ for SU(3-6) gauge theories, plotted against $T_{c} / T$. For SU(3), we have used the plaquette data of Ref. [5].

The pressure was calculated using the so-called integral method [5], Eq.(1.1), which determines $p(T)$ in terms of pressure at some reference temperature $p\left(T_{0}\right)$. We find that $p(T) \sim 0$ within our errors till temperatures $\sim 0.9 T_{c}$. This is probably related to the fact that glueballs are much heavier than $T_{c}$, and therefore are not excited substantially except very close to $T_{c}$. We therefore evaluate $p / T^{4}$ by taking $\beta_{0}\left(T<0.8 T_{c}\right)$ as the lower limit of the integral, with the knowledge that the omitted additive constant is smaller than the statistical error.
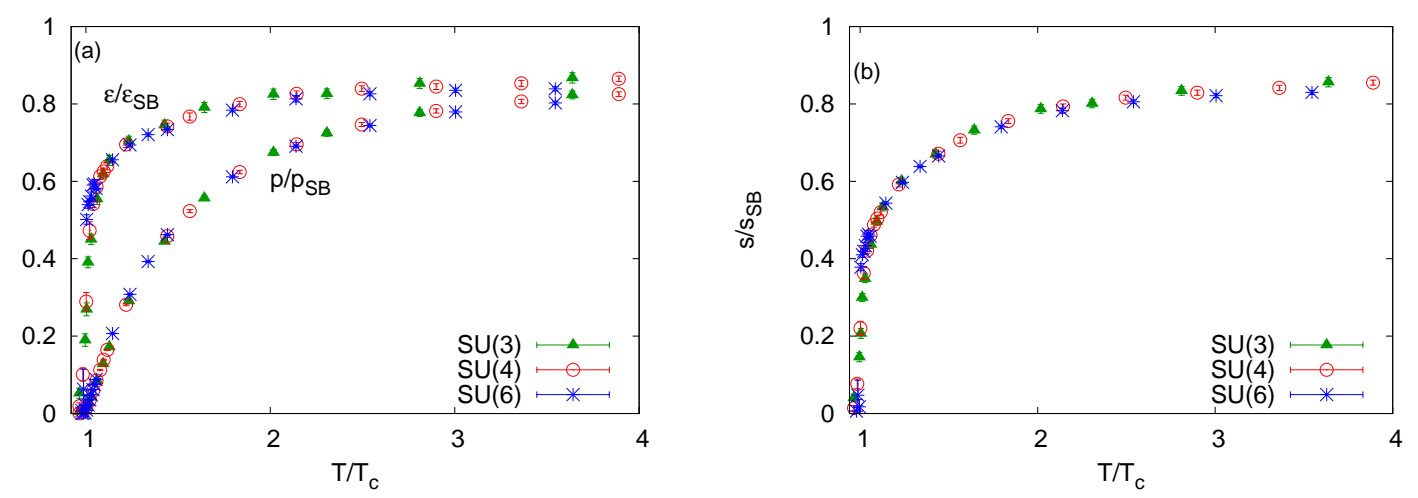

Figure 3: (a) The pressure and energy density for $\mathrm{SU}(\mathrm{N})$ gauge theories with $\mathrm{N}=3,4$ and 6 , normalized to their Stefan-Boltzman values. (b) The same for the entropy density.

Other bulk thermodynamic quantities can be obtained from a knowledge of $p(T)$ and $\Delta(T)$. Figure 3 shows a summary of our results for energy density, pressure and entropy density for SU(N) gauge theories, normalized to their Stefan-Boltzman limits. The Stefan-Boltzman values used are the results calculated for the free theory on the lattice, for the integral method [13]:

$$
\frac{\varepsilon_{S B}}{T^{4}}=3 \frac{p_{S B}}{T^{4}}=\frac{\pi^{2} d_{A}}{15} G\left(N_{t}\right) \quad \text { where } \quad G\left(N_{t}\right)=1+\frac{8 \pi^{2}}{21} \frac{1}{N_{t}^{2}}+\cdots
$$


As the figures show, even at temperatures close to $4 T_{c}$, one reaches $\sim 85 \%$ or less of the StefanBoltzman value. The figures also show that, plotted as a function of temperature, the size of the $1 / \mathrm{N}^{2}$ corrections is small in all the three observables, except for very close to $T_{c}$ where $1 / \mathrm{N}^{2}$ corrections are visible in the energy density and entropy, as expected from the sizable $1 / \mathrm{N}^{2}$ correction in the latent heat, Eq. (2.3).

\section{Summary and Discussions:}

We have presented results for bulk thermodynamic quantities for $\mathrm{SU}(\mathrm{N})$ gauge theories with $\mathrm{N}=3,4$ and 6. Our results for latent heat of the transition and its dependence on $\mathrm{N}$ are summarized in Table 1 and Eq. (2.3). We find that the latent heat for the theory with three colors is substantially less than that with larger number of colors: the size of the leading correction in Eq. (2.3) is substantial for $\mathrm{N}=3$.

The bulk thermodynamic quantities are shown in Fig. 3. It is found that even at temperatures close to $4 T_{c}$, one reaches only $\sim 85 \%$ of the Stefan-Boltzman value. We also see that except very close to $T_{c}$, the size of the leading $1 / \mathrm{N}^{2}$ correction is small, when we compare the bulk thermodynamic quantities measured at the same value of $T / T_{c}$. Interestingly, the scaling with the number of colors is better when looked at as function of $T / T_{C}$ than when considered as function of the 't Hooft coupling. Figure 4 (a) shows the entropy density plotted against the 't Hooft coupling, defined through the $\mathrm{V}$ scheme and evaluated at the scale $2 \pi T$, where the running is done through our nonperturbative beta function. Considerable $1 / \mathrm{N}^{2}$ correction is now seen, in particular for $T<1.5 T_{c}$. Of course, the scaling with $\lambda$ will be different depending on the scheme chosen to define the coupling. The figure also shows $s / s_{S B}$ for the $\mathscr{N}=4$ supersymmetric theory [4]. This theory is seen to have deviations from the Stefan-Boltzmann value of the same size as pure $\mathrm{SU}(\mathrm{N})$ theory at the highest temperatures, but a very different dependence on $\lambda$.
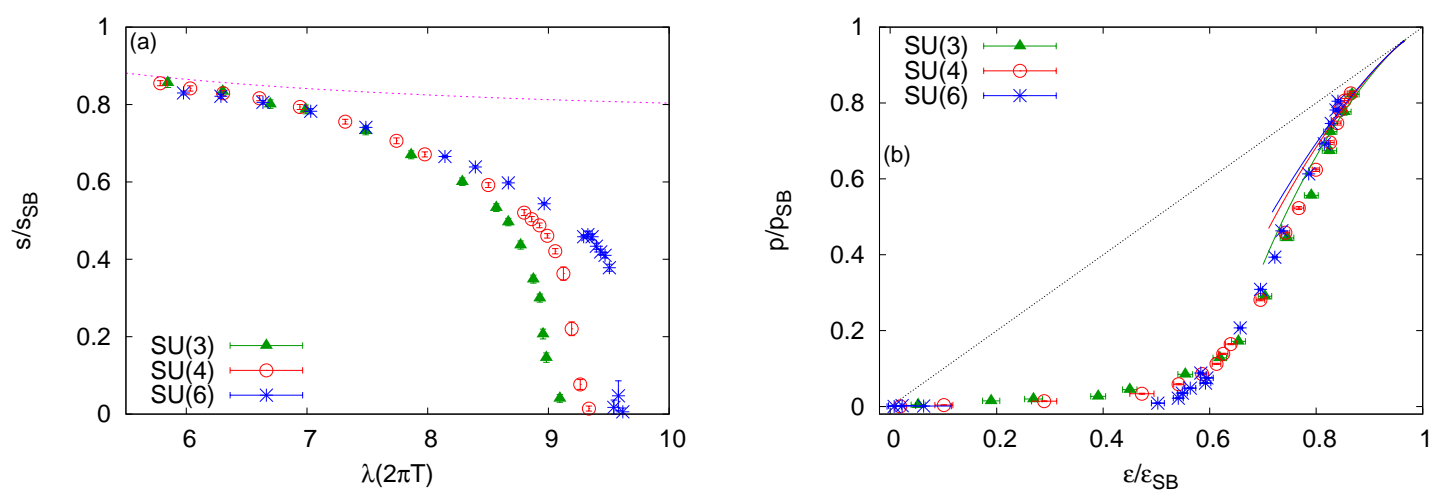

Figure 4: (a) Entropy density, in units of the Stefan-Boltzman value, shown as a function of the 'tHooft coupling. The pink dotted line is the result for the $\mathscr{N}=4$ supersymmetric $\mathrm{SU}(\mathrm{N})$ theory for large $\mathrm{N}$ [4]. (b) $p / p_{S B}$ shown as a function of $\varepsilon / \varepsilon_{S B}$. The diagonal line is the line of conformal theories while, by construction, the Stefan-Boltzman limit is the point $(1,1)$. The weak coupling results for the different theories are also shown.

The large deviation of the thermodynamic quantities from the Stefan-Boltzmann value even at $4 T_{c}$, and the fact that some strongly coupled conformal field theories show similar deviations 
from Stefan-Boltzmann limit, have been used in the literature to speculate about a strongly coupled, conformal regime in pure $\mathrm{SU}(\mathrm{N})$ gauge theories. The thermodynamics results can be used to investigate the feasibility of such a phase. Following Ref. [14], in Fig. 4 (b) we plot the energy density vs. pressure, normalized by the corresponding Stefan-Boltzmann values. By construction the point at $(1,1)$ is the Stefan-Boltzmann limit, while the diagonal line denotes conformality. Also shown are the weak coupling lines for the theories with the different number of colors [15]. We find that for the temperature regime of interest to RHIC, $\sim 2 T_{c}$, the theory is far from conformal. At higher temperatures, the theory approaches conformality, but it is closer to the weak coupling line than the conformal line, indicating the absence of a strongly coupled, conformal phase in the $\mathrm{SU}(\mathrm{N})$ gluon plasma.

The computations were carried out on the workstation farm of the department of theoretical physics, TIFR. We thank Ajay Salve for technical support.

\section{References}

[1] G. 't Hooft, Nucl. Phys. B 72 (1974) 461.

[2] S. Datta and S. Gupta, Continuum Thermodynamics of the GluoN $N_{c}$ Plasma. arXiv:1006.0938.

[3] R. Pisarski and L. McLerran, Nucl. Phys. A 796 (2007) 83.

[4] S. S. Gubser, I. R. Klebanov and A. A. Tseytlin, Nucl. Phys. B 534 (1998) 202.

[5] G. Boyd et al., Nucl. Phys. B 469 (1996) 419.

[6] S. Datta and S. Gupta, Phys. Rev. D 80 (2009) 114504.

[7] R. V. Gavai, Nucl. Phys., B 633 (2002) 127.

[8] B. Lucini, M. Teper and U. Wenger, Phys. Lett., B 545 (2002) 197; J. H. E. P., 0401 (2004) 061. B. Lucini and M. Teper, J. H. E. P. 0502 (2005) 033.

[9] B. Bringoltz and M. Teper, Phys. Lett., B 628 (2005) 113.

[10] M. Panero, Phys. Rev. Lett. 103 (2009) 232001.

[11] S. Datta and S. Gupta, Properties of the $S U\left(N_{c}\right)$ Gluon Plasma. in proceedings of Lattice 2009, POS (LAT2009) 178.

[12] R. Pisarski, Prog. Theor. Phys. Suppl., 168 (2007) 276.

[13] J. Engels, F. Karsch and T. Scheideler, Nucl. Phys. B 564 (2000) 303.

[14] R. V. Gavai, S. Gupta and S. Mukherjee, Pramana 71 (2008) 487.

[15] M. Laine and Y. Schröder, Phys.Rev. D 73 (2006) 085009. 